\title{
Preliminary Experimental Study on Aerodynamic Characteristics Control of Slender Body Using DBD Plasma Actuator
}

\author{
By Hiroyuki NishidA ${ }^{1)}$, Sakae MizUKI ${ }^{2)}$, Isao MiYAZAKI ${ }^{1)}$, Satoshi NONAKA ${ }^{2)}$, Taku NonOMURA ${ }^{3)}$ \\ and Yoshifumi INATANI ${ }^{3)}$ \\ 1) Department of Mechanical Systems Engineering, Tokyo University of Agriculture and Technology, Koganei, Japan \\ ${ }^{2)}$ Department of Aeronautics and Astronautics, University of Tokyo, Tokyo, Japan \\ ${ }^{3)}$ The Institute of Space and Astronautical Science, Japan Aerospace Exploration Agency, Sagamihara, Japan
}

(Received June 25th, 2011)

\begin{abstract}
Asymmetric separation vortices over a slender body at a high angle of attack exert a strong side force on the body and lead to the loss of attitude stability. We investigated the active control of the separation flow over a slender body and addressed the proportional control of the side force and the pitching moment. A flow control experiment was conducted in a wind tunnel using a cone-cylinder test body and a Dielectric Barrier Discharge (DBD) plasma actuator as a flow control device. The free-stream velocity was $9 \mathrm{~m} / \mathrm{s}$ and the Reynolds number was approximately 42000 . The side force coefficient was proportionally controlled within approximately \pm 1.0 using the actuator at the aft body, and the static stability angle of attack was controlled from 25 to 40 degrees and 65 to 85 degrees by controlling the pitching moment when the center of gravity was at the $55 \%$ position from the body apex. We estimated that a higher actuator output power is required for the effective control of the aerodynamics in a real flight. In addition, we confirmed that the actuator burst operation mode could reduce the required output power.
\end{abstract}

Keywords: Flow Control, DBD Plasma Actuator, Slender Body

\section{Nomenclature}

$\begin{array}{lll}C_{y} & : & \text { side force coefficient } \\ C_{\phi} & : & \text { actuator control force coefficient } \\ D & : & \text { model base diameter or burst ratio } \\ f+ & : & \text { burst frequency } \\ F_{y} & : & \text { side force } \\ F_{a c} & : & \text { actuator thrust force } \\ F_{D B D} & : & \text { actuator thrust force per unit length } \\ L & : & \text { total length of the test model } \\ L_{c o n e} & : & \text { length of the cone } \\ L_{c y l i n d e r} & : & \text { length of the cylinder } \\ L_{D B D} & : & \text { length of the plasma actuator } \\ q & : & \text { dynamic pressure of the free-stream } \\ R_{N} & : & \text { curvature radius of the nose tip } \\ S & : & \text { base area of the test model } \\ T & : & \text { burst cycle } \\ T_{o n} & : & \text { duty cycle } \\ T_{P M} & : & \text { pitching moment } \\ \theta_{c o n e} & : & \text { nose apex angle }\end{array}$

\section{Introduction}

A slender body is a typical body shape of a rocket vehicle or a fore body of an aircraft. In general, it is known that asymmetric separation vortices occur over a slender body when the angle of attack becomes two times higher than the fore body apex angle ${ }^{1)}$, and these asymmetric separation vortices exert side forces and yawing moments on the body that cause a disturbance in the attitude stability.

Characteristics of the flow field over a slender body can be classified into four groups according to the apex angle of the body and the angle of attack (see Fig. 1) ${ }^{1}$.

(1) When the angle of attack is sufficiently low, the axial flow is dominant in the flow around the body (the cross flow around the body is much weaker than the axial flow), and flow separation does not occur. The schematic of this flow type is shown in Fig. 1 (1).

(2) The cross flow becomes stronger with a higher angle of attack and separates at the side surface. The vortex filaments are shed from the sides of the apex and transported downstream along the body while the vortex structure remains symmetric. The schematic of this flow type is shown in Fig. 1 (2), and $\alpha_{\mathrm{SV}}$ [Remark 2]= $1.1-1.3 \theta_{\text {cone. }}$

(3) When the angle of attack exceeds the threshold value, the vortex filaments break away from the body surface and the vortex structure becomes asymmetric. The asymmetricvortices exert strong side forces on the body. The schematic of this flow type is shown in Fig. 1 (3), and $\alpha_{\mathrm{AV}}=2 \theta_{\text {cone }}$.

(4) This flow field is similar to that over a two-dimensional cylinder and vortices are periodically shed. The schematic of this flow type is shown in Fig. 1 (4), and $\alpha_{\mathrm{UV}}=70-75$ degrees. 


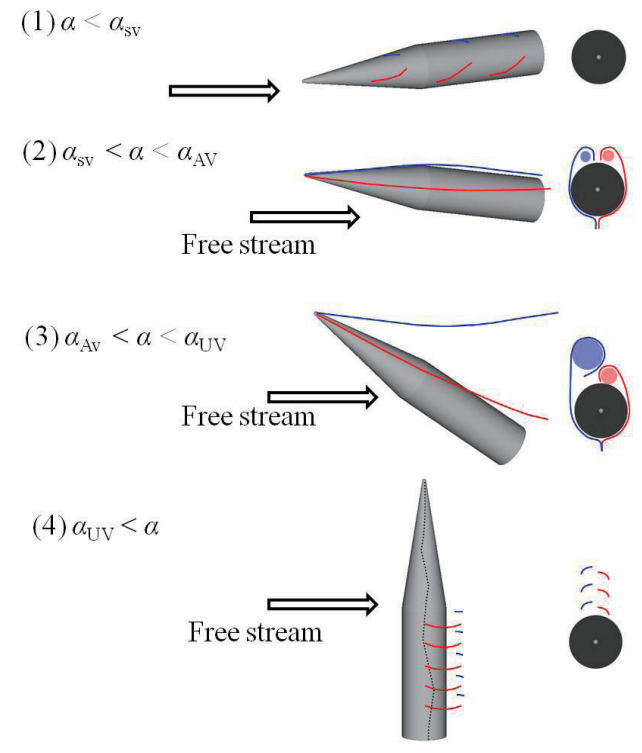

Fig. 1. Characteristics of the flow field over a slender body ${ }^{1)}$.

Many studies have been conducted trying to clarify the generation mechanism of the asymmetric separation vortices and understand the structure and transport characteristics of these vortices (these topics are reviewed in references ${ }^{2,3)}$ ). These studies aim at the suppression and control of the asymmetric vortices (i.e., the side forces) using flow-control devices because conventional attitude-control devices such as thrusters and rudders are inefficient and/or ineffective. High-power thrusters are required because the large side force amplitudes and rudders in the wake flow do not work very well. An active-flow control is one of the effective solutions, and several active-flow control devices such as micro jets and small moving flaps have already been proposed and studied $^{2-5)}$; however, no active-flow control device has been put into practice because of its wide-ranging mechanics and/or additional high-pressure working fluids.

In recent years, a flow-control method using a Dielectric Barrier Discharge (DBD) plasma actuator received much attention for its effectiveness ${ }^{6-8)}$. The DBD plasma actuator has a simple configuration as shown in Fig. 2. It consists of two electrodes separated by a dielectric and is displaced in the streamwise direction. An alternating voltage applied to the electrode induces the ionization and generates plasma. The discharge causes flow acceleration along the dielectric surface away from the exposed electrode (see Fig. 2). Momentum is added to the boundary layer by setting the actuator on the body surface, and the flow separation can be suppressed. Although the applied voltages are high $(\sim 20 \mathrm{kV})$, the electric currents $(\sim 0.1 \mathrm{~A})$ and the required electric power remain low. This is a practical advantage of a plasma actuator. The other advantages are that this is an electronic device without any moving parts, has fast response, and is thin and light in weight. There exist some studies where a plasma actuator acts as a control device for the asymmetric vortices over a slender body ${ }^{9}{ }^{10}$. The plasma actuator was installed on the nose tip of the slender body and only a bang-bang control of the side force was accomplished (In general, it is known that a control effect at the nose tip is too sensitive for the asymmetric vortex structure to proportionally control the side force ${ }^{2)}$ ). However, the plasma actuator can be easily installed anywhere on the body surface. It is thought that the proportional control of the side forces and the pitching moment is possible by multipoint actuator installations on the body surface.

In this paper, we attempt to verify the proportional control concept of the aerodynamic characteristics. and report the results of a preliminary experiment on the aerodynamic characteristics control using a cone-cylinder shaped test model and a DBD plasma actuator in a low-speed wind tunnel.

In this study, we considered two types of the operational modes - continuous and burst mode — of the plasma actuator .

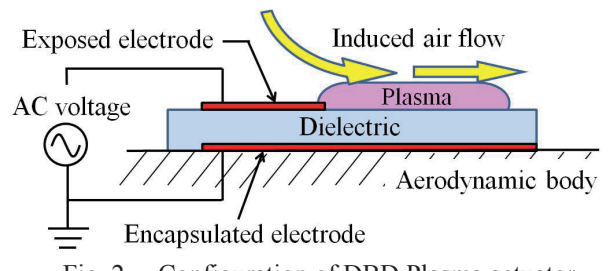

\section{Experimental Setup and Procedures}

\subsection{DBD plasma actuator}

The DBD plasma actuator consists of two electrodes and a dielectric as shown in Fig. 2. The electrodes and the dielectric are made of a $0.06 \mathrm{~mm}$ thick copper tape and $0.8 \mathrm{~mm}$ thick Teflon sheet, respectively.

Two types of electric power supply systems are used to apply an alternating voltage to the exposed electrode. One type is an electric power supply for the ozone production (LHV-13AC) that can provide a quasi-sinusoidal voltage wave with a peak value of up to $12 \mathrm{kV}$ and a frequency of $5.5 \mathrm{kHz}$. The other type is a high-speed electric power amplifier (HEOPT-5B20) that can amplify any voltage wave with a frequency of up to $100 \mathrm{kHz}$. The maximum peak value of the output voltage wave is up to $5 \mathrm{kV}$ and the input voltage wave is generated by a function generator.

The DBD plasma actuator produces a thrust force, which is measured with an electronic balance and defined as the DBD plasma actuator controlling force, as the reaction force to the induced flow momentum. Figure 3 shows the thrust measurement system; the thrust stand is inside a vacuum chamber, and the plasma actuator is mounted on the electronic balance. In Fig. 4, the thrust force is plotted as a function of the applied voltage the measured result of the thrust force is shown. For convenience, the thrust force is normalized to the free-stream momentum flux as follows:

$$
C_{\phi}=\frac{F_{a c}}{q S}=\frac{F_{D B D} L_{D B D}}{q S} .
$$




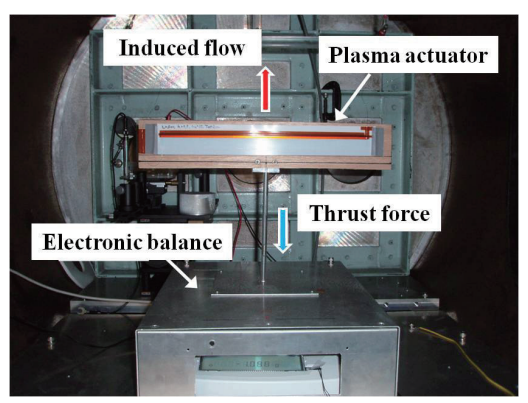

Fig. 3. Thrust measurement system of DBD plasma actuator.

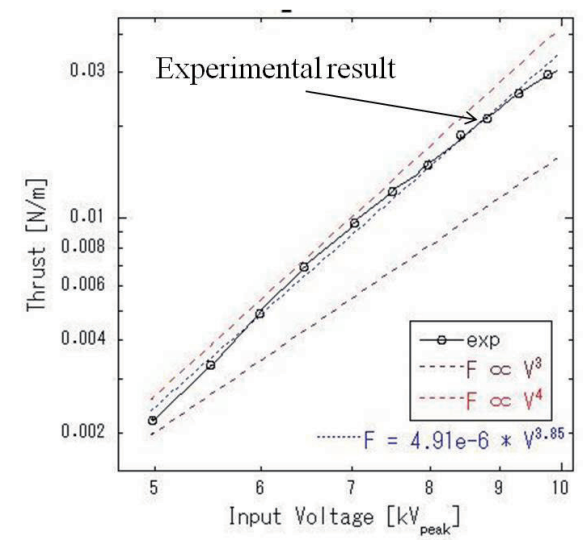

Fig. 4. Thrust force of DBD plasma actuator as a function of applied voltage.

\subsection{Wind-tunnel test model}

The shape of the wind-tunnel test model is shown in Fig. 5. It consists of a cone and a cylinder, and its dimensions are summarized in Table 1. In this study, the plasma actuator is attached along the body axis (the induced flow by the actuator is in the circumferential direction). The upper and lower half of Fig. 6 shows the plasma actuators and the test model equipped with the plasma actuators, respectively.

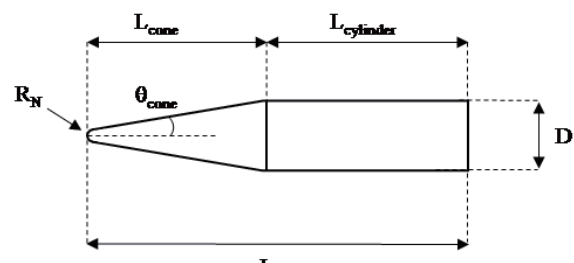

Fig. 5. Configuratioth of DBD plasma actuator.

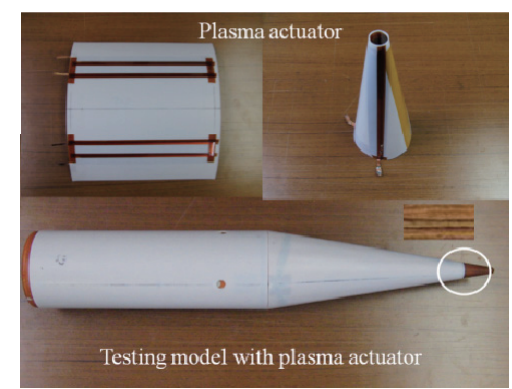

Fig. 6. Configuration of DBD plasma actuator.
Table 1. Dimensions of the test model.

\begin{tabular}{|c|c|}
\hline$L$ & $0.421[\mathrm{~m}]$ \\
\hline$L_{\text {cone }}$ & $0.211[\mathrm{~m}]$ \\
\hline$L_{\text {cylinder }}$ & $0.210[\mathrm{~m}]$ \\
\hline$D$ & $0.072[\mathrm{~m}]$ \\
\hline$R_{\mathrm{N}}$ & $0.0028[\mathrm{~m}]$ \\
\hline$S$ & $0.00407\left[\mathrm{~m}^{2}\right]$ \\
\hline$\theta_{\text {cone }}$ & $9[\mathrm{deg}]$ \\
\hline $2 R_{\mathrm{N}} / D$ & 0.079 \\
\hline Slender Ratio & 5.85 \\
\hline
\end{tabular}

\subsection{Wind-tunnel experiment}

These experiments are conducted in a low-speed wind tunnel at ISAS/JAXA. The flow speed ranges from $7 \mathrm{~m} / \mathrm{s}$ to $50 \mathrm{~m} / \mathrm{s}$.

The angle of attack of the test model is controlled by a turning table installed in the wind tunnel. The aerodynamic force and moment acting on the test model are measured by an internal balance, which has six components, and the cross flow of the test model is visualized by the two-dimensional Particle-Image-Velocimetry (PIV) method. The setting schematics of the PIV system are shown in Fig. 7. One $\mu \mathrm{m}$ diameter oil particles are introduced in the free-stream and are visualized by a double pulse Nd-YAG laser. The time averaged flow field is obtained by averaging 300 pictures.

\subsection{Experimental conditions}

In all experiments, the free-stream velocity is $9 \mathrm{~m} / \mathrm{s}$ and the Reynolds number is about 42000; the flow is in the laminar region. The angle of attack of the test model ranges from 0 to 90 degrees.

Two types of plasma actuator operations are used: the continuous and burst operation modes. In the continuous operation mode, the plasma actuator is driven by a continuous voltage wave supplied by the electric power supply for the ozone production (LHV-13AC), whereas in the burst operation mode, it is driven by an unsteady input voltage known as "duty cycle" or "burst wave" as illustrated in Fig. 6. The burst wave is generated by a function generator and amplified by a high-speed electric power amplifier (HEOPT-5B20). The burst ratio $\left(\mathrm{D}=T_{\mathrm{on}} / T\right)$ and the burst frequency $(f+=1 / T)$ are operational parameters. To select the operational parameters, an experiment is conducted using a three-dimensional cylinder; the shape of this cylinder is same as that of the test model, and the actuator is circumferentially attached at +80 degrees from the stagnation point (only at one side). The lift force control experiment is conducted with various burst frequencies $(30-600 \mathrm{~Hz})$, constant applied voltage $(5 \mathrm{kV}$ peak), and constant duty ratio $(10 \%$ or $50 \%)$. The effective burst frequency is surveyed for each duty ratio. In Fig. 9, the change in the lift force coefficient $\left(\Delta C_{y}\right)$ is plotted as a function of the burst frequency $(f+)$ and the experimental results are shown. This study aims to analyze the flow-control effect of the burst operation, and therefore only the most effective burst frequency in each duty ratio is adopted according to the experimental result of the three-dimensional cylinder; (burst ratio, burst frequency) $=(10 \%, 200 \mathrm{~Hz})$ and $(50 \%, 600 \mathrm{~Hz})$. These experiments were conducted in both the operational modes with various thrust forces, $C \phi$. 


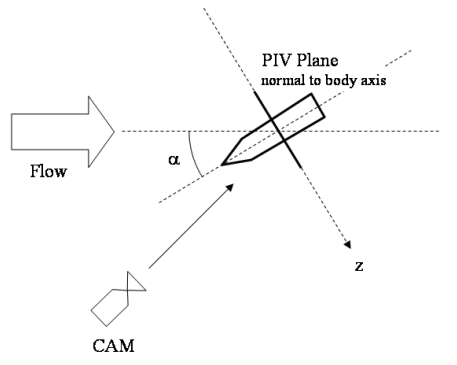

Fig. 7. Schematic of PIV measurement system.

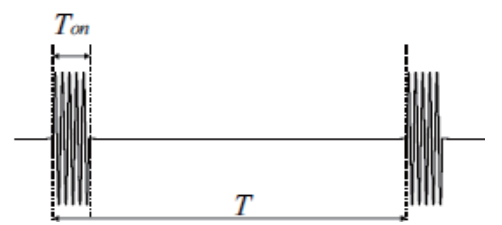

Fig. 8. Unsteady duty cycle wave.

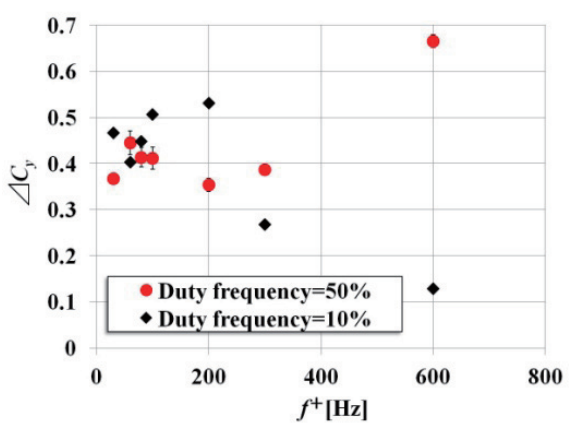

Fig. 9. Lift force control experiment of a three-dimensional cylinder in burst operation mode.

\section{Pitching Moment Control}

First, we show the experimental results of the pitching moment control, in which the plasma actuator operation mode is continuous.

Figure 10 shows the installation locations of the actuators; the actuators are circumferentially located at \pm 80 degrees from the stagnation point. These actuators are operated only at the fore (cone) or aft (cylinder) body (see Fig. 10) in two operational types: the forward and backward operations. In these operations, the airflow is induced in the forward and backward directions against the cross flow. It is thought that the forward operation suppresses the flow separation while backward operation promotes flow separation.

Figure 11 shows the pitching moment coefficient as a function of the angle of attack; the gravity center is assumed at position $x / L=0.55$ from the body apex and the positive pitching moment indicates the pitch-up moment. The pitching moment coefficient is defined as follows:

$$
C_{m}=\frac{T_{P M}}{q S D} .
$$

The error bars in the actuator-off case are from the standard deviation of several experiments, and those in the actuator-on cases are estimated based on the deviations of the actuator thrust (average 10 percent). The pitching moment characteristics for the actuator-off case are compared with those for the actuator-on cases; the $C \phi$ of the actuator is 0.056 . It can be observed from Fig.11 that the pitching moment is changed by the plasma actuator operation in all operation cases. When the forward operation at the fore body suppresses the flow separation, the drag force acting on the fore body decreases, and as a result, a pitch-down moment acts on the test body. On the other hand, in the case of the forward operation at the aft body, the drag force acting on the aft body decreases, and a pitch-up moment acts on the test body. When the flow separation is promoted by the backward operation of the plasma actuator, the drag force increases at the operational actuator position, and the pitching moment responds to the change in the drag force.

It can be confirmed from Fig. 11 that the stable angle of attack, where the body attitude is statically stable, can be controlled from 25 to 40 degrees and 65 to 85 degrees. Of course, this controllability depends on the position of the gravity center .

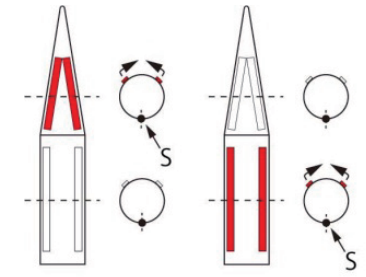

Forward operation

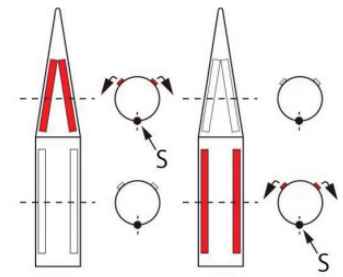

Backward operation
Fig. 10. Positions and operational procedures of the plasma actuators for the control of the pitching moment.

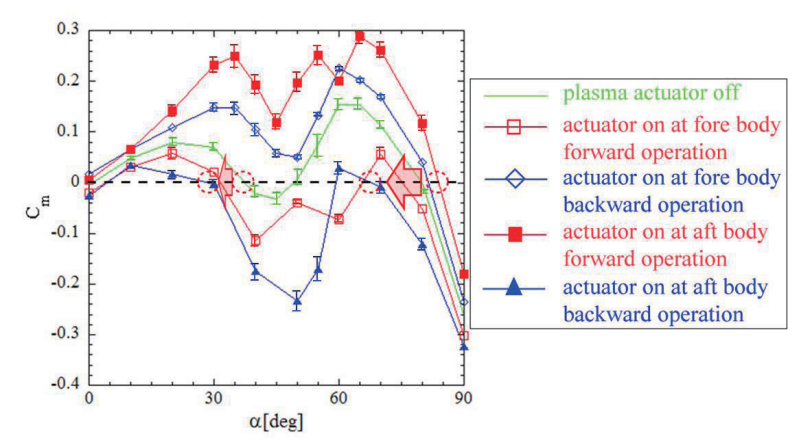

Fig. 11. Pitching moment coefficient as a function of the angle of attack for various plasma actuator operations $(C \phi=0.056)$.

\section{Side Force Control}

\subsection{Control in continuous operation mode}

As mentioned earlier, in some studies the plasma actuator has been installed on the nose tip of a slender body. However, only a bang-bang control of the side force has been attempted and it has been decided that the proportional control is difficult due to the bistable characteristics of the asymmetric vortices. In this study, we attempt to proportionally control the side force with the plasma actuator at the aft body.

Figure 12 shows the installation locations of the plasma actuators, which are circumferentially located at \pm 80 degrees from the stagnation point. Black arrows in Fig. 12 indicate the actuator induced flow directions.

In Fig. 13, the side force coefficient is plotted as a function 
of the angle of attack; the green, blue, and red lines indicate the cases in which all actuators are off, the port-side actuator is on, and the starboard actuator is on. The thrust force of the plasma actuator, $C \phi$, is 0.028 and the positive value of the side force means the rightward side force vector (see the red arrow in Fig. 12). The side force coefficient is defined as follows:

$$
C_{Y}=\frac{F_{Y}}{q S} .
$$

Figure 13 shows that the side force reaches a maximum for an angle of attack of approximately 50 degrees. As shown in Fig. 13, the side force is changed by the plasma actuator. It increases in the direction to the operating actuator; the rightward side force increases when the starboard actuator is on whereas the leftward side force increases when the port-side actuator is on. The mechanism that causes the side force to change by the plasma actuator is discussed using the flow visualization result. Figure 14 shows the cross-flow field obtained by the PIV measurement in the case of an angle of attack of 60 degrees; the vorticity distributions and streamlines are also shown in this figure. The left- and right-hand side of Fig. 14 show the cross-flow field with actuator-off and the port-side actuator-on, respectively (the red arrows indicate the direction of the induced flow by the plasma actuator). The asymmetric vortices can be clearly observed in Fig. 14. It can be seen that the cross-flow separation is slightly suppressed by the actuator operation type and the flow above the operating actuator is deflected leftward. It is thought that the side force change by the actuator is due to the flow separation suppression. However, only a slight flow field deformation is observed in Fig. 14 despite the large side force change $\left(\Delta C_{y} \sim 2\right)$. A more detailed flow field information (more precise flow visualization or numerical flow field simulation) is needed to understand the side force control mechanism better.

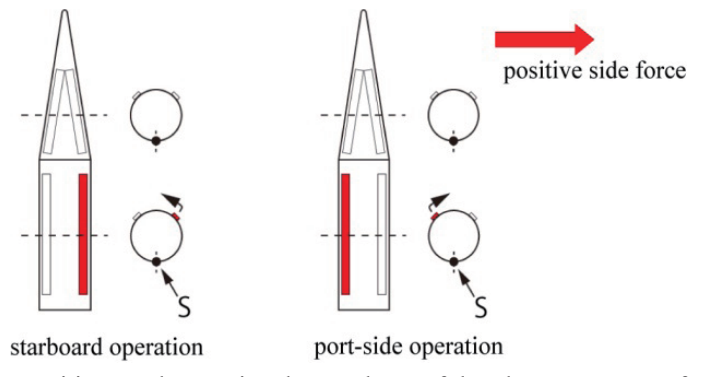

Fig. 12. Positions and operational procedures of the plasma actuators for the side force control.

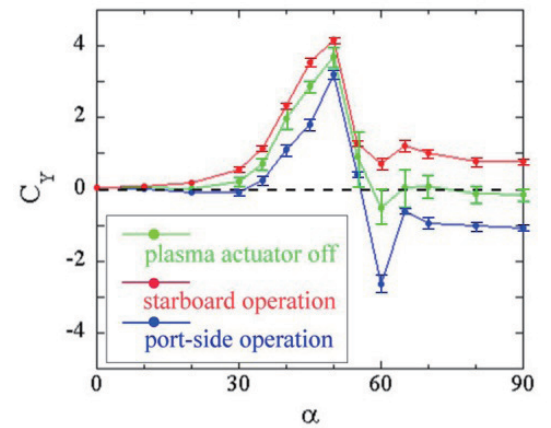

Fig. 13. Side force coefficient plotted against the angle of attack.

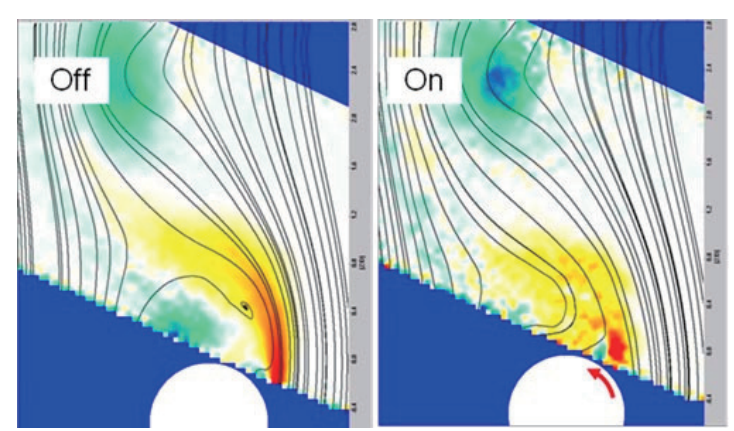

Fig. 14. PIV measurement of the cross-flow field behind the test model at the angle of attack of 60 degrees; the cross-flow plane at $\mathrm{x} / D=4.5$ from the body apex. The vorticity distributions and streamlines are also shown.

Figure 15 plots the relation between $\Delta C_{y}$ and $C \phi$ for the angles of attack of $40,50,60$, and 70 degrees. Here, $\Delta C_{y}$ is the side force change caused by the flow control, and the positive and negative $C \phi$ indicate the starboard and port-side actuator operation, respectively. Although the sensitivity to the controlling power $C \phi$ depends on the angle of attack, it is observed that the side force can be proportionally controlled as a function of $C \phi$. Although the side force coefficient can be controlled within \pm 1.0 on an average, a higher actuator power is required to make the side force zero at the angles of attack of 50 and 60 degrees.

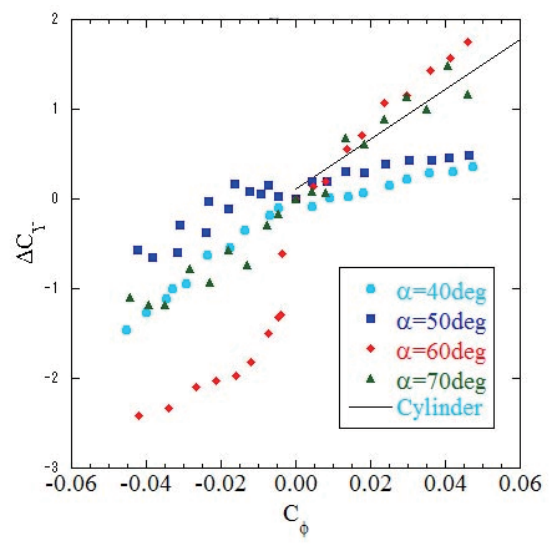

Fig. 15. Side force coefficient plotted against the angle of attack.

From the above results, the aerodynamic characteristics control concept is verified using a DBD plasma actuator. However, a higher actuator thrust force is needed for a higher flow control effect. In this experiment, the free-stream velocity is $9 \mathrm{~m} / \mathrm{s}$. In a real flight with a free-stream velocity of more than $100 \mathrm{~m} / \mathrm{s}$, the required thrust force $(\mathrm{N} / \mathrm{m})$ of the plasma actuator is estimated to be very high. The current level of technical development of the DBD plasma actuator does not allow to generate even $1 \mathrm{~N} / \mathrm{m}$. Therefore, it is necessary to reduce the required thrust force by optimizing the installation location and the operation mode of the DBD plasma actuator.

\subsection{Control in burst operation mode}

As discussed above, the required thrust force needed for a real application is not within current capabilities. In recent 
years, the flow control in the burst operation mode of the plasma actuator has received much attention for its effectiveness $^{7-10)}$. This burst mode periodically generates a large vortex whose size is larger than the boundary layer. The periodically shed vortex causes a mixture between the free-stream and the boundary layer, and momentum is transported from the free-stream to the boundary layer. This mixture leads to effective flow control.

In this section, the burst operation mode of the plasma actuator is applied to the control of the side force at a high angle of attack, and its effectiveness is evaluated. The experimental setup is the same as that in section 5.1.

Figure 16 shows the flow control effect on the side force $\left(\Delta C_{y}\right)$ plotted as a function of the thrust force of the actuator $(C \phi)$, and the comparison between the continuous and burst operation mode is shown in this figure. In the burst operation mode, the base voltage wave is the sinusoidal wave of $9 \mathrm{kHz}$, and the burst ratio (D) and burst frequency $(f+)$ are $50 \%$ and $600 \mathrm{~Hz}$, or $10 \%$ and $250 \mathrm{~Hz}$. Although a significant control effect cannot be observed in both cases of the continuous and burst operation modes in Figs. 16 (a) and (b) (note, however, that $C \phi$ is quite low due to the limitation of the electric power supply by the high-speed electric power amplifier), it can be observed in Figs. 16 (c) and (d) that the burst operation mode can generate a higher flow-control effect even with a lower thrust force. The highest flow-control effect can be obtained in the case of a $10 \%$ burst ratio.

This preliminary experiment shows the possibility that the burst operation mode of the actuator reduces the required thrust force for the control of the flow field over a slender body. Although it seems that the proportional controllability is lost with the burst operation mode, the experiment in this study was conducted in a small range of $C \phi$. Therefore, further studies with varying $C \phi$ in a wide range are needed to evaluate the proportional controllability of the burst operation mode.

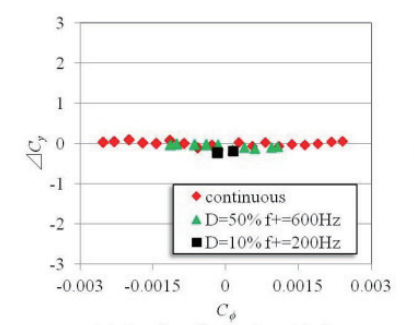

(a) Angle of attack $=40 \mathrm{degs}$.

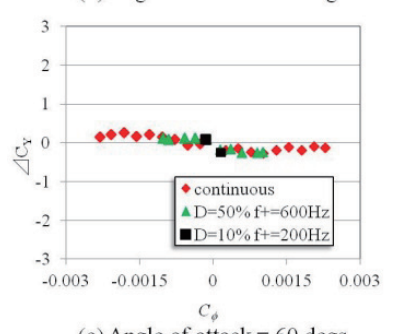

(c) Angle of attack $=60 \mathrm{degs}$.

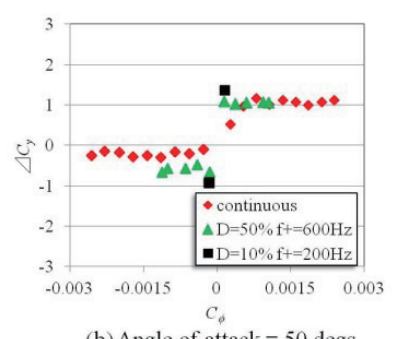

(b) Angle of attack $=50$ degs.

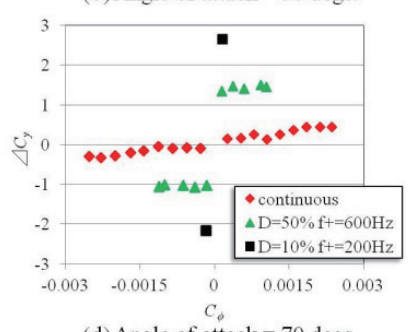

(d) Angle of attack $=70 \mathrm{degs}$.
Fig. 16. Change in the side force by the actuator operation; comparison between the continuous and burst modes.

\subsection{Discussion}

It can be observed in Figs. 15 and 16 that the controllability of the aerodynamic force strongly depends on the angle of attack. In addition, the flow-control effect with a starboard actuator operation is quite different from that with a port-side actuator operation. These comprehensive relations among the flow control effect, the installation locations of the actuator, and the angle of attack are due to the comprehensive structure of the separation flow and the nonlinear behavior of the separation vortices in response to the angle of attack.

A detailed three-dimensional structure of the separation flow with the plasma actuator operation must be clarified to optimize the installation location and the operation method of the plasma actuator. Further studies using numerical simulation and detailed flow visualization are indispensable.

\section{Concluding Remarks}

We studied the active control of the separation flow over a slender body and addressed the proportional control of the side force and the pitching moment. A flow control experiment was conducted in a wind tunnel using a cone-cylinder test body and a DBD plasma actuator as a flow control device. The pitching moment can be controlled by operating the plasma actuators only at the fore or aft body. In this study, the static stability angle was controlled from 25 to 40 degrees and 65 to 85 degrees by controlling the pitching moment when the center of gravity was at the $55 \%$ position from the body apex. The side force coefficient was proportionally controlled to within approximately \pm 1.0 while operating the actuators at the aft body. These experiments showed the possibility that the aerodynamic characteristics of a slender body at a high angle of attack can be proportionally controlled by the plasma actuator. In addition, a higher actuator thrust force is needed in a real application. It was confirmed that the burst operation mode of the plasma actuator could reduce the required thrust force. However, the controllability of the aerodynamic force strongly depends on the angle of attack and the operational location of the actuator. The comprehensive relations among the flow control effect, the installation locations of the actuator, and the angle of attack are due to the comprehensive structure of the separation flow and the nonlinear behavior of the separation vortices against the angle of attack. The detailed three-dimensional structure of a separation flow with a plasma actuator operation must be clarified to optimize the installation locations and the operation method of the plasma actuator. Further studies using numerical simulation and detailed flow visualization are indispensable.

\section{Acknowledgments}

This research was partly supported by KAKENHI; Grant-in-Aid for Research Activity Start-up (21860028). The authors would like to thank Enago for the English language review. 


\section{References}

1) Ericsson, L.E. and Reding, J.P.: Steady and Unsteady Vortex-Induced Asymmetric Loads on Slender Vehicles, J. Spacecraft and Rockets, 18 (1981), pp.97-109.

2) David H. B.: The Asymmetric Vortex Wake Problem - Asking the Right Question, AIAA Paper 2006-3553, 2006.

3) Hunt, B. L.: Asymmetric Vortex Forces Wakes on Slender Bodies, AIAA Paper19 82-1336, 1982.

4) Ptel, M.P. , Tilmann, C.P. and Ng, T. T.: Closed-Loop Missile Yaw Control via Manipulation of Forebody Flow Asymmetries, Journal of Spacecraft and Rockets, 41 (2004), pp.436-443.

5) Bernhardt, J.E. and Williams, D.R.: Proportinal Control of Asymmetric Forebody Vortices, AIAA J., 36 (1998), pp.2087-2093.

6) Post, M.L. and Corke, T.C.: "Separation Control on High Angle of Attack Airfoil using Plasma Actuator",AIAA
Journal. 42. 2177 (2004).

7) Tsubakino, D. and Tanaka, K.: Effective Layout of Plasma Actuators for a Flow Separation Control on a Wing, AIAA Paper 2007-474, 2007.

8) Asada, K, Fujii, K.: Computational Analysis of Unsteady Flow-field Induced by Plasma Actuator in Burst Mode, AIAA Paper 2010-5090, 2010.

9) Liu, F., Luo, S., Gao, C., Meng, X., Hao, J., Wang, J. and Zhao, Z.: Flow Control over a Conical Forebody Using Duty-Cycled Plasma Actuators, AIAA J., 46 (2008).

10) Matsuno, T., Kawazoe, H., Nelson, R. C. and Corke, T. C.: Forebody Vortex Control on High Performance Aircraft Using PWM-Controlled Plasma Actuators, Proceedings of the $26^{\text {th }}$ ICAS Congress, 2008. 